\title{
A TEST FOR PRESENCE OR ABSENCE OF PROANTHOCYANIDINS IN BARLEY AND MALT
}

\author{
by \\ STEN AASTRUP \\ Department of Brewing Chemistry, Carlsberg Research Laboratory, \\ Gamle Carlsberg Vej 10, DK-2500 Copenhagen Valby
}

Keywords: Anthocyanogens, vanillin test, testa

\begin{abstract}
An unambiguous method for distinguishing proanthocyanidin-free barley grains from proanthocyanidin-containing ones is described. Sanded surfacs of grains are stained with a vanillin- $\mathrm{HCl}$ solution. The testa of grains containing proanthocyanidines turns red while proanthocyanidin-free grains remain uncoloured. Four hundred grains can be prepared in five minutes and stained in 30 minutes.
\end{abstract}

\section{INTRODUCTION}

Since it was first published by vON WETTSTEIN et al. (11) that a proanthocyanidin-free barley mutant (ant 13-13) gave rise to beer with excellent haze stability, intensive breeding work has been carried out to obtain improved lines $(8$, $10,12,13,14)$ and these were used to study the role of proanthocyanidins in malting and brewing $(3,5,6,14)$.

The breeding carried out in Europe, North America, Japan and New Zealand has resulted in more than 400 additional proanthocyanidinfree mutants in 65 spring and 9 winter barley varieties and a wide selection of recombinant lines (14). Thereby proanthocyanidin-free barley lines not suffering from the severe yield reduction and mildew susceptibility characteristic for the original mutant (ant 13-13), have been obtained. Lines selected from crosses of ant 13-13 with Rupal and Nordal, and most especially the mutant in Triumph, ant 17-148 (named Galant) have competitive yielding ability and satisfactory agronomic performance (2, 14).

Pilot and production scale malting and brewing with proanthocyanidin-free barleys have produced malt and beer which satisfy the prescribed specifications (14). Taste panel evaluations demonstrated that the improved colloidal stability was not associated with alterations in flavour and flavour stability $(3,5,6)$.

The results of the malting and brewing experiments together with the improvements of agronomic performance of the proanthocyanidin-free barleys make their commercial use realistic. Therefore it was desirable to improve the method so far employed for discriminating grains with and without proanthocyanidins (12) in such a way that an instantaneous control of the purity of proanthocyanidin-free lots of barley or malt becomes feasible at the breeding stations, at the seed testing stations, at the elevators of malt houses and in the breweries.

\section{MATERIALS AND METHODS}

\subsection{Plant material}

\subsubsection{Mixtures}

Four lots ( 3000 grains each) of proanthocyanidin-free barley samples containing increasing admixtures of proanthocyanidin-containing grains were made up by mixing counted grains of 

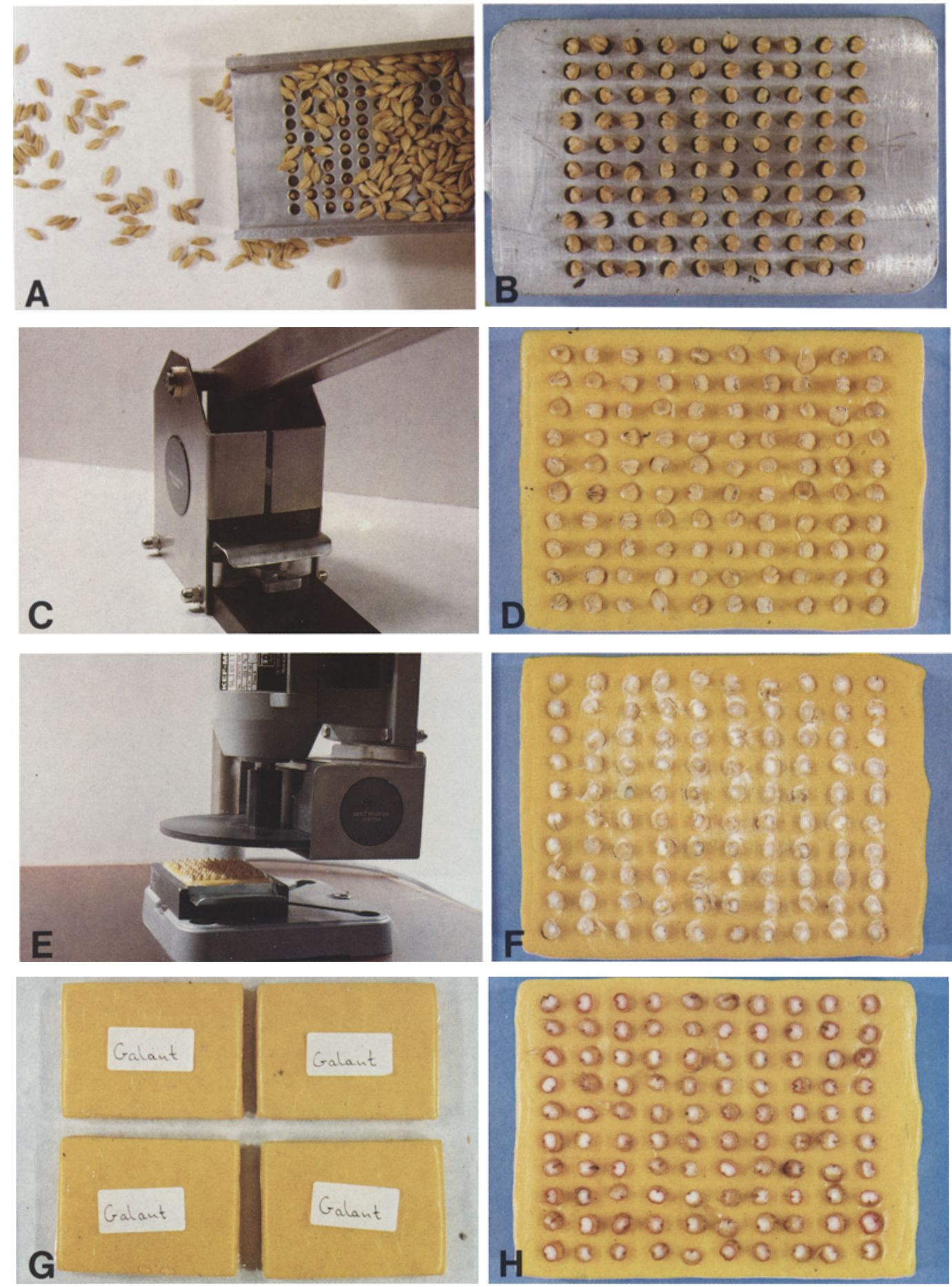
Figure 1. Pictorial description for testing if barley grains or malt kernels contain proanthocyanidins.

From a sample of 200 to 400 grains, 100 grains are oriented by placing them on an overmatrix (A) and shaking them into position in the undermatrix (B). The undermatrix with the grains in position is placed in the press unit (C) and the grains pressed into thermoplastic clay, Cernit (D).

A disc sander $(E)$ is used for removing one quarter of each of the grains $(F)$.

The sanded grains are stained for half an hour in a vanillin- $\mathrm{HCl}$ solution. Four Cernit blocks placed upside down with the sanded grains submerged in the staining solution is shown $(\mathrm{G})$. After staining $(\mathrm{H})$ the proanthocyanidincontaining grains show a distinct red ring while no red colour is observed in the proanthocyanidin-free grains.

ant 13-13 with counted Triumph kernels. The following lots were made:

Lot A: $1 \%$ proanthocyanidin-containing grains.

Lot B: $5 \%$ proanthocyanidin-containing grains.

Lot $\mathrm{C}: 10 \%$ proanthocyanidin-containing grains.

Lot D: $30 \%$ proanthocyanidin-containinggrains.

\subsubsection{Field propagations}

Samples from three propagation plots of $\mathrm{Ga}$ lant grown at three different farms in Denmark were obtained. Three samples of breeding lines
(Galant (ant 17-148) $\times$ Keti, ant 13-13 × Rupal, ant 5022(mutant in Sonja)) were from plots at the Carlsberg Experimental Farms. Ant 13-13× Rupal was malted for testing the corresponding malt kernels. All barley samples were supplied by Carlsberg Plant Breeding.

\subsection{Sample preparation}

For staining the grains are prepared using the Carlsberg Seed Fixation System (7, 9) (Danbrew Consult Ltd., Rahbeks Alle 21, DK-1801

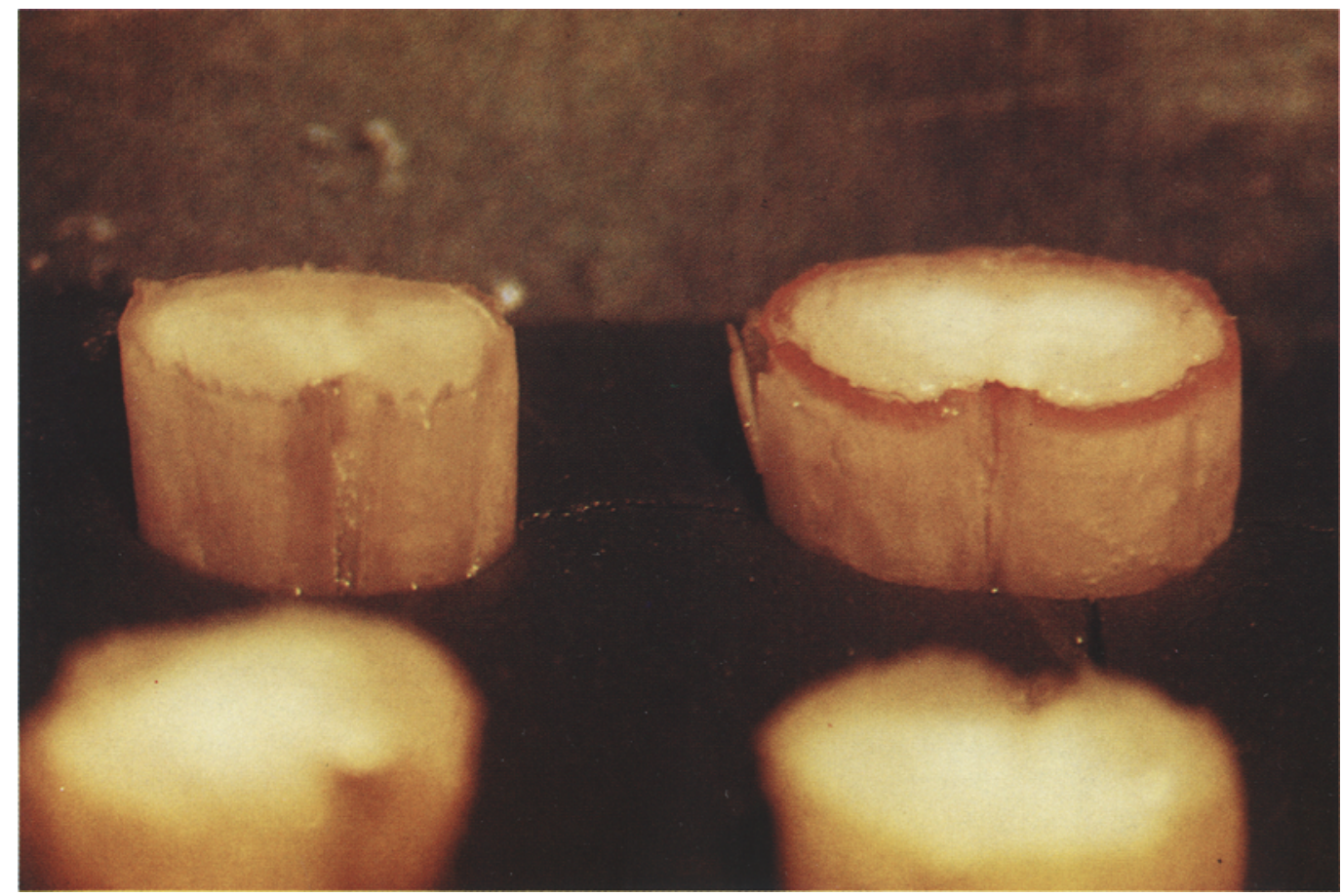

Figure 2. A detail of a plate showing a vanillin- $\mathrm{HCl}$ stained proanthocyanidin-free grain (left) and a vanillin- $\mathrm{HCl}$ stained proanthocyanidin-containing grain (right). 
Copenhagen V, Denmark) as follows:

1) From a representative sample of 200 to 400 grains, 100 grains are placed into the undermatrix containing cylindrico-conical depressions using an overmatrix with round holes. (Figures $1 \mathrm{~A}$ and $\mathrm{B}$ ). The depressions in the undermatrix have a depth which covers half the grain.

2) The grains are fixed in a block of thermoplastic clay, Cernit ( $\mathrm{T}+\mathrm{F} \mathrm{GmbH}$, Rosenaustrasse 9, D-6072 Dreieich, W. Germany) by means of a specially designed press (Figures $1 \mathrm{C}$ and D) in such a way that the half of each grain which rises above the undermatrix is embedded in the Cernit.

3) One quarter of each grain, that is half of the part of each grain which rises above the Cernit, is removed using a disc sander (Figures $1 \mathrm{E}$ and $\mathrm{F}$ ).

\subsection{Staining}

The proanthocyanidins are rendered visible by staining the sanded grains with a freshly prepared $1 \%$ vanillin- $6 \mathrm{M}-\mathrm{HCl}$ solution (1). The Cernit blocks are placed in the solution for half an hour (Figure $1 \mathrm{G}$ ). A distinct red colour develops in the testa of the proanthocyanidincontaining grains, while the proanthocyanidinfree grains lack the red colour (Figure $1 \mathrm{H}$ and Figure 2). The colour remains stable for at least half an hour.

\section{RESULTS AND DISCUSSION}

\subsection{Sample size}

Proanthocyanidin-free barley varieties as other cultivars are pure lines. After propagation admixtures of less than $1 \%$ proanthocyanidincontaining grains are desirable, the contamination resulting from mechanical admixtures or outcrossing. At the elevators of grain storage facilities or malthouses the contamination of a proanthocyanidin-free barley line or a lot should be determinable with an accuracy of a few $\%$. Towards this aim an examination of 400 grains ( 4 plates) has been chosen.

\subsection{Use of equipment and reading of results}

Preparing the samples by use of the Carlsberg Seed Fixation System, supplied with a matrix specially made for this method as described under 2.2. and Figure 1 ensures an easy, rapid and safe fixation of the grains. The total working time for a sample of 400 grains (four plates) is about 5 minutes.

The sanding procedure, after which a portion of the grains still rises above the Cernit after the operation, in combination with the vanillin $-\mathrm{HCl}$ staining technique, ensures an easy access to the staining solution and reading of the results by eye or with a magnifying glass. A distinct red colour turns up in the testa of the proanthocyanidin-containing grains while the proanthocyanidin-free grains do not stain. The "red ring" of the cross-sectioned grain is also visible through the palea, lemma and pericarp (husks) in the upper $1 \mathrm{~mm}$ of the sanded grain. The optimal position for reading the results is looking at the plates at an angle of $15^{\circ}$ (Figure 2 ). The reading should be completed within half an hour as the red colour fades and the grains become black with time.

The result is given as percentage of grains containing proanthocyanidins.

\subsection{Reproducibility and reliability of the method}

Using 400 grains ( 4 plates) per analysis the method was evaluated on four artificially made mixtures $\mathrm{A}, \mathrm{B}, \mathrm{C}$ and $\mathrm{D}$ having $1 \%, 5 \%, 10 \%$ and $30 \%$ proanthocyanidin-containing grains, respectively.

The result of five successive analyses of the individual samples (Table I) showed values very close to the true values.

Five independent analyses were carried out on samples from a plot of a proanthocyanidin-free barley breeding line which revealed an admixture of proanthocyanidin-containing grains, and compared with the analysis of malt derived from this line (Table II). A high reproducibility is demonstrated, and as expected no significant differences in the percentage of admixture is seen between the barley sample and the malt made from it.

Determinations of admixture percentages of 
Table I

Five successive determinations of the percentage of proanthocyanidin-containing barley grains with the vanillin test in samples constructed by mixing increasing proportions of Triumph kernels with ant 13-13 proanthocyanidin-free kernels. For each determination $\mathbf{4 0 0}$ kernels were removed from the sample totaling 3000 grains.

\begin{tabular}{lrrrrrr}
\hline $\begin{array}{l}\text { Constructed } \\
\text { sample } \\
\text { (\% Triumph }\end{array}$ & I & II & III & IV & V & I - V \\
in ant 13-13) & & & & & & \\
\hline A “1\%" & 1.3 & 1.0 & 1.3 & 0.5 & 1.3 & 1.1 \\
B " 5\%" & 5.3 & 5.8 & 5.8 & 4.5 & 4.8 & 5.2 \\
C “10\%" & 10.8 & 9.5 & 10.3 & 10.5 & 8.9 & 10.0 \\
D"30\%" & 32.5 & 29.8 & 29.5 & 29.5 & 29.8 & 30.2 \\
\hline
\end{tabular}

\section{Table II}

Comparison of the percentage of proanthocyanidin-containing grains in the plot of a proanthocyanidin-free barley breeding line (ant 13-13 $\times$ Rupal) and the malt prepared from it. For both samples five independent determinations with the vanillin test were performed on $\mathbf{4 0 0}$ kernels fixed and sanded in Cernit clay.

\begin{tabular}{llllllll}
\hline Sample & I & II & III & IV & V & Mean & S.D. \\
\hline Barley & 5.8 & 5.5 & 4.8 & 5.8 & 5.3 & 5.4 & 0.42 \\
Malt & 5.0 & 5.3 & 5.1 & 5.4 & 5.8 & 5.3 & 0.31 \\
\hline
\end{tabular}

\section{Table III}

Determination of proanthocyanidin-containing admixtures in propagation fields with the vanillin test on kernels fixed and sanded in Cernit clay.

\begin{tabular}{lccc}
\hline Variety/Line & $\begin{array}{c}\text { Number of proanthocanidin- } \\
\text { containing grains in a } \\
\text { sample of } 400\end{array}$ & $\begin{array}{c}\text { Admixture } \\
(\%)\end{array}$ & $\begin{array}{c}95 \% \text { confidence } \\
\text { limits (4) }\end{array}$ \\
\hline Galant & 1 & 0.3 & $0.01-1.38$ \\
Galant & 23 & 5.8 & $3.68-8.51$ \\
Galant & 2 & 0.5 & $0.06-1.79$ \\
Galant $\times$ Keti & 17 & 4.3 & $2.49-6.73$ \\
Ant 5022 & 0 & 0 & $0.00-0.92$ \\
\hline
\end{tabular}

larger field propagations (Table III) demonstrate that the method is suitable for checking propagation plots for admixtures. Three of the five large scale propagations show a very low frequency of proanthocyanidin-containing grains. This underscores the observation that all mutants and recombinant lines exhibiting the proanthocyanidin-free trait have been found genetically stable (14). In instances where it was possible to trace the source of admixtures it was found to be due to mechanical admixtures or spontaneous outcrossing. 


\section{CONCLUSION}

Vanillin- $\mathrm{HCl}$ staining of flour from single grains (12) has been successfully applied for the identification of a large number of proanthocyanidin-free mutants, recombinant lines and for checking purity of the breeding material. By combining the results of the histochemical location of proanthocyanidins in the testa (1) with those obtained with the seed fixation system ( 7 , 9) it was possible to develop a method which permits the detection of proanthocyanidincontaining contaminants in 400 "proanthocyanidin-free" kernels in 5 minutes. This satisfies the requirements for checking the purity of proanthocyanidin-free barley lines in seed testing, large scale propagation, grain trade, malt production and malt trade.

\section{ACKNOWLEDGEMENTS}

The author wishes to thank Professor D. vON WetTStein, Research Manager B. AhrenstLARSEN and K. ERDAL for help in preparing the manuscript. The skilful technical assistance of Mss Ella Meiling, LiLlemor Bank FredeRIKSEN and NANCY GAMMELBY is highly appreciated.

\section{REFERENCES}

1. Aastrup, S., H. OutTrup \& K. Erdal: Location of the proanthocyanidins in the barley grain. Carlsberg Res. Commun. 49, 105-109 (1984)

2. CRISP. J: Triumph mutant gives encouraging results. Brewers' Guardian, June 1984, 21 -22 (1984)

3. Delcour, J.A., M.M. VANDEnberghe, P. DonDEYNe, E.L. SChrevens, J. WiJnhoven \& E. MOERMAN: Flavour and haze stability differences in unhopped and hopped all-malt pilsner beers brewed with proanthocyanidin-free and with regular malt. J. Inst. Brew. 90, 67-72 (1984)

4. Documenta Geigy: Mathematics and statistics, pp. 98-103, CIBA-GEIGY Limited, Basel, Switzerland (1975)

5. Erdal, K., B. Ahrenst-Larsen \& B. JendeSTRID: Use of proanthocyanidin-free barley in beer brewing. In Cereals for Food and Beverages. G.E. Inglett \& L. Munck eds. Academic Press, New York, pp. 365-379 (1980)

6. Erdal, K., H. OutTrup \& B. Ahrenst-Larsen: The role of proanthocyanidins in beer flavour and flavour stability. Proc. Europ. Brewery Conv. Congr., London, S57-564 (1983)

7. Heltved, F., S. Aastrup, O. Jensen, G. Gibbons \& L. MUNCK: Preparation of seeds for mass screening. Carlsberg Res. Commun. 47, 291-296 (1982)

8. INGVERSEN, J., J. LARSEN \& K. ERdal: Breeding of proanthocyanidin-free malting barley. Barley Newsletter 26, 72-74 (1983)

9. JeNSEN, S. AA. \& S. AASTRUP: Determination of malt modification. Cerevisia (1985) in press

10. LARSEN, J.: Breeding of proanthocyanidin-free malt barley. In: Barley Genetics IV, Edinburgh, pp. $211.216(1981)$

11. Wettstein, D. von, B. Jende-Strid, B. AhRENST-LARSEN \& J.A. Sørensen: Biochemical mutant in barley renders chemical stabilization of beer superfluous. Carlsberg Res. Commun. 42, 341-351 (1977)

12. Wettstein, D. von, B. Jende-Strid, B. AhRENST-LARSEN \& K. ERDAL: Proanthocyanidinfree barley prevents the formation of beer haze. MBAA Tech. Quarterly 17, 16-23 (1980)

13. Wettstein, D. von, J. LaRsen, B. Jende-Strid, B. Ahrenst-Larsen. J.A. Sørensen \& K. ERdal: Proanthocyanidin-freie Braugersten. Brauwelt 121, 760-767 (1980)

14. Wettstein. D. von, R.A. Nilan, B. AhrenstLarsen, K. Erdal, J. IngVersen, B. JendeStRID, K. NyegaARd KristiansEn, J. LARSEN, H. OUTTRUP \& S.E. UlLRICH: Proanthocyanidin-free barley for brewing: Progress in breeding for high yield and research tool in polyphenol chemistry. MBAA Tech. Quart. 22 (1985) in press

Accepted by: H. KLENOW, E. LUND and S.O. ANDERSEN 